Revue européenne des sciences sociales

European Journal of Social Sciences

XXXIX-121 | 2001

L'acteur. Un concept sur la scène des sciences sociales

\title{
L'acteur. Une notion en question dans les sciences sociales
}

Jean Pierre Gaudin

\section{OpenEdition}

Journals

Édition électronique

URL : http://journals.openedition.org/ress/641

DOI : $10.4000 /$ ress. 641

ISSN : 1663-4446

Éditeur

Librairie Droz

Édition imprimée

Date de publication : 1 novembre 2001

Pagination : 7-14

ISBN : 2-600-00663-X

ISSN : 0048-8046

Référence électronique

Jean Pierre Gaudin, «L'acteur. Une notion en question dans les sciences sociales », Revue européenne des sciences sociales [En ligne], XXXIX-121 | 2001, mis en ligne le 11 décembre 2009, consulté le 10 décembre 2020. URL : http://journals.openedition.org/ress/641 ; DOI : https://doi.org/10.4000/ress. 641 
Jean Pierre GAUDIN

\section{L'ACTEUR UNE NOTION EN QUESTION DANS LES SCIENCES SOCIALES}

Une multiplicité de significations se trouve attachée au mot l'acteur ainsi qu'au terme auquel il s'est progressivement substitué en français: le comédien. A la scène ou à l'écran, ce personnage se caractérise, en effet, par le respect d'une contrainte, celle de suivre un texte et/ou de respecter une situation, mais également par une marge non négligeable d'initiative, d'invention, en somme de jeu.

Notons ensuite qu'on ne trouve guère, semble-t-il, dans la littérature scientifique anglo-saxonne de correspondance directe au terme acteur: fort peu d'actor (quelques occurrences très récentes toutefois en sociologie), mais plutôt diverses sémantisations qui renvoient plus directement à des domaines constitués des sciences sociales (par exemple the individual, pour la sociologie des organisations; the self, pour la psychologie; ou bien même des substantifs de remplacement comme behaviors, pour le approches comportementales, de type comportementaliste ou stratégique; ou encore interaction, en sociologie ou psychologie sociale).

Dans la littérature des sciences sociales de langue française, du moins, l'usage intensif et la plasticité du terme, sa valeur métaphorique et ses ambivalences, autorisent à la fois quelques passerelles entre disciplines mais également toutes les ambiguités...

L'acteur, est devenu un «mot de passe» dans les sciences sociales mais sans qu'il soit forcément réservé aux initiés qui en connaissent le sens précis. Dans le usages courants et le sens commun, la référence à l'acteur social suggère surtout un peu vaguement la liberté d'initiative, mais aussi les ressources du comportement rationnel et, pour tout dire, s'associe à la figure de l'individu moderne.

Dans les sciences sociales et humaines, les constructions de la notion s'avèrent très diverses et les usages multiformes, même en première analyse. Si la philosophie, pour sa part, s'est essentiellement appuyée sur la distinction sujet/ objet, il est possible de distinguer différentes approches, le sujet pouvant s'apparenter à l'individu ou au contraire s'en différencier fortement, l'autonomie du sujet étant alors posée comme autre que la liberté ou la singularité de l'individu. Dans certains courants disciplinaires des sciences sociales, on peut aussi avancer de manière volontairement lapidaire que la notion d'acteur s'apparente par ses usages à celles de sujet (en droit, en psychologie) ou d'individu rationnel (théorie standard, élargie ou non, en économie où les firmes et les syndicats d'intérêts sont ramenées d'ailleurs à des logiques d'individus; sociologie des organisations dans ses versions initiales des années cinquante; problématique du choix rationnel et théorie des jeux appliquées en science politique, par exemple). Tandis que dans d'autres approches, d'inspiration plus structurale, on parlera volontiers d'agents, 
inscrits dans des contextes ou bien des contraintes explicatives de leurs actes (macro-économie, sociologies structuralistes, etc).

Dues pour partie à la croissante spécialisation des sciences mais, plus encore peut-être, aux clivages épistémologiques qui, en traversant les disciplines, apparentent des choix méthodologiques au delà des frontières convenues des savoirs, il y a eu ainsi dans les sciences sociales des réceptions très différentiées des termes acteur et agent, dont on a voulu rechercher les linéaments.

On peut ainsi relever que la mobilisation relativement récente de la catégorie d'acteur s'est inscrite dans trois enjeux scientifiques majeurs.

Celui qui s'attache à l'objectivation de ce qui est observé, en premier lieu. Autrement dit, l'acteur social est-il par définition le seul élément susceptible d'une analyse, ou bien est-il possible (et judicieux) au cours de la construction et du déroulement de l'analyse de donner ce même statut d'acteur (pris dans un ensemble de stratégies et de systèmes de valeurs) tant à l'observateur qu'à l'observé? Les conséquences d'un tel choix seront évidemment importantes quant au processus d'observation, au traitement de l'information et à la nature des résultats attendus.

Celui, ensuite, des articulations entre actions individuelles et collectives. Comment l'acteur social (qu'il soit individuel ou bien de type collectif) peut-il être situé, entre autonomie et contraintes sociales? Si l'on cherche à caractériser quelles sont les logiques de ses pratiques et actions, on sera conduit à tenter d'élaborer des formes de méso-analyse pour explorer les interactions entre les individus et la société entière.

Celui, enfin, du choix des cheminements méthodologiques, enjeu qui est d'ailleurs difficilement dissociable du précédent. En d'autres termes, fera-t-on le pari de rechercher les explications de manière agrégative, partant du particulier pour espérer aller au plus général? Ou bien tentera-t-on, en cheminant dans l'autre sens en quelque sorte, d'interpréter principalement en fonction d'une conception holiste de la société les places, fonctions et rôles des acteurs sociaux? Là encore l'utilisation ou non de la notion d'acteur, la place qu'on lui donnera dans la caractérisation des phénomènes observés apparaissent lourdes de conséquences.

Ces différents enjeux d'analyse se trouvent en pratique souvent étroitement mélés dans le processus de construction scientifique de la réalité. Afin de ne pas sous-estimer les liens qui les unissent, c'est donc en selon une perspective plus transversale qu'on présentera ici quelques réflexions introductives: en examinant pourquoi et comment la catégorie d'acteur a pu tenir une place croissante dans l'explicitation des relations individus -société; mais également en s'interrogeant sur l'ampleur de ce «retour de l'acteur», au regard des formes de légitimation contemporaines des sciences sociales.

\section{L'ANALYSE DES RELATIONS INDIVIDUS-SOCIÉTÉ}

\section{a. Les dualismes épistémologiques, leur efficacité et leurs limites}

Un certain nombre de savoirs normatifs et de démarches d'analyse se sont initialement constitués à partir de l'observation et de la problématisation de situa- 
tions individuelles ou inter-individuelles (tels les prérogatives du propriétaire ou bien le droit de la personne, dans le premier code civil; le comportement rationnel de l'homo economicus chez les économistes marginalistes, ou encore les caractéristiques de l'habitant pour les démographes et la Statistique nationale). Cette perspective d'ailleurs n'excluait pas intellectuellement d'envisager des agrégations de ces situations.

Mais d'autres savoirs ont d'emblée plutôt cherché à construire l'analyse à partir d'ensembles collectifs ou de contextes sociétaux, dotés par hypothèse de logiques propres. Ce qui dans cette approche n'empêche pas de faire place, cependant, aux interactions entre ces cadres sociaux et les pratiques (ou autonomies) individuelles; c'est ce que tentent les conceptualisation de la notion de «milieu» chez les géographes classiques, de celle de «société» chez les premiers sociologues durkheimiens, les psychologues et criminologues de la même génération, ou préalablement de celle de «nation» pour la majorité des historiens du XIX"

Cependant, l'ambition compréhensive va s'avérer en pratique plus durablement (et plus facilement) affichée que pratiquée. Selon qu'on s'attachera plutôt au trait général ou au signe particulier, et qu'on prétendra mettre en valeur explicative la détermination ou à l'inverse la contingence, on aura quelque tendance à perdre de vue les interactions les plus heuristiques; ou, pire, on les instrumentera, pour en définitive les hiérarchiser dans un sens ou l'autre de manière un peu mécaniste. Dans le sens d'un privilège donné aux problématiques «micro » (échelle de l'individu), la stratégie de l'agent économique (y compris la firme ou le syndicat collectif d'intérêts) est ramenée chez les économistes standard à un semblant de comportement individuel rationnel, dans une grande continuité avec les antécédents de la discipline; tandis que dans l'autre sens, celui qui donne l'avantage aux problématiques macro (échelle de la société entière), la démarche historique envisagée par l'école des Annales en rupture avec les courants événementialistes du $\mathrm{XIX}^{\mathrm{e}}$ siècle sera bientôt rivée à la vie matérielle collective et aux échanges économiques, et ce jusqu'à l'aggiornamento partiel que représentera l'histoire des «mentalités » à partir des années 60.

La réflexion scientifique a pu ainsi longuement se couler au cours de ce dernier siècle dans des dualismes, explicites ou non, qui avaient pour effet de dichotomiser échelles micro et macro, individu et société, liberté et contrainte. Le risque de réduction était d'ailleurs particulièrement grand lorsque les termes de ces différents dualismes se conjuguaient entre eux, assimilant l'acteur, l'individu et l'idée de liberté par exemple.

A plusieurs reprises, cependant, des remises en cause de ces raisonnements dichotomiques ont été entreprises, facilitées en particulier (mais ce n'est pas la seule raison) par l'affaiblissement du positivisme qui avait constitué le socle épistémologique commun du premier développement des sciences sociales au XIX ${ }^{\mathrm{e}}$ et au début du $\mathrm{XX}^{\mathrm{e}}$.

\section{b. De quelques voies de renouveau méthodologique et de la fortune consécutive de «l'acteur» dans les problématiques contemporaines des sciences sociales}

Parmi les voies tendant à réarticuler individu et société qui ont pu être explorées, trois d'entre elles ont apporté dans les sciences sociales un renouveau, même s'il est inégal selon les disciplines. 
Les problématiques systémiques, au premier chef.

Elles sont sans doute peu spécifiques par rapport à notre interrogation relative à l'acteur, mais ce sont les mieux susceptibles d'applications générales et les plus anciennes au regard de la critique des explications scientifiques de type linéaire et positiviste. Elles ont en particulier permis de développer de nouvelles analyses de l'action. En sociologie de organisations et en analyse de la décision, leur apport peut apparaître double. Le raisonnement systémique force, d'un côté, à tenir compte de l'environnement du système et donc à contextualiser les approches relationnelles, à en penser les feed-back avec l'extérieur et donc à concevoir des boucles explicatives entre les initiatives individuelles et les cadres sociaux. Ce type de raisonnement conduit, par ailleurs, à envisager chaque acteur-en-interaction dans des situations sociales concrètes, faites non pas de transparence pure et parfaite, comme dans les problématiques du choix rationnel, mais caractérisées par de l'information éventuellement partielle, et où les acteurs sont mus par une rationalité, elle aussi, limitée (H. Simon). C'est là une façon d'appréhender ainsi, comme un tout, les rapports individu-société et liberté-contrainte, même si elle fait souvent l'impasse sur les systèmes de valeurs qui sont en jeu et sur les positions sociales des acteurs. En tout état de cause, l'analyse reste, ce faisant, dans un cadre de raisonnement contraint par son actionnalisme même (il serait plus valorisant d'être acteur qu'agi)?

Les approches cognitives de l'action, autre tentative intellectuelle de réarticulation à laquelle on pense alors, semblent, pour leur part, échapper à ce caractère normatif.

L'action est là encore un point de départ central de la réflexion. Pour A. Schütz, il s'agit de conduites humaines, prévisibles à l'avance et associées à des motifs et des état de conscience. L'acteur (ici essentiellement individuel) devient alors inséparable de la notion de projet personnel. Néanmoins dans pareille optique, l'interprétation du monde par l'acteur n'apparaît possible, dans le même temps, que sur la base d'une réserve sociale d'expériences préalables, c'est à dire sur un stock de connaissances historiquement constitué. A la suite de Schütz, les démarches des cognitivistes américains des années 60 ont tenté d'aller encore de l'avant dans la remise en cause des dichotomies traditionnelles, en s'affranchissant d'une perspective initiale qui restait assez micro-sociologique. La prise en compte d'entités plus larges que les individus et leurs interactions directes (ou de face-à-face) s'est alors orientée vers les institutions, organisations, réseaux relationnels, et normes sociales, qui apparaissent comme «formatant» au moins partiellement les activités individuelles de construction du monde social.

Ce faisant, nous sommes alors conduits à interroger le statut de l'acteur dans un voisinage intellectuel également très renouvelé mais mouvant, celui de ce qu'on appelle aujourd'hui les constructivismes sociaux.

Il s'agit là de la réunion un peu arbitraire et floue, comme tous les étiquetages d'écoles, de démarches qui partageraient la préoccupation essentielle de saisir tout à la fois les processus d'intériorisation de l'objectif et d'extériorisation du subjectif dans différents mondes sociaux. Le pari intellectuel est que la réalité n'est ni pure objectivité ni seulement représentation. Les réalités sociales sont donc envisagées en tant que «construits » historiques et quotidiens des acteurs individuels et collectifs. Par sa démarche historique, N. Elias s'est en particulier interrogé sur l'apparition du moi dans le monde moderne, comme conscience de 
soi et comme être pensant; plus largement, il a voulu caractériser le statut contemporain de la personnalité et de l'individu dans la pensée scientifique. A partir de la métaphore du jeu, il a envisagé la société comme un ensemble de dépendances réciproques, reliant entre eux les individus. L'accent mis sur l'interdépendance plutôt que sur l'interaction entend souligner fortement que les relations ne sont pas nécessairement équilibrées ou égales et qu'il faut faire une place centrale dans les analyses d'acteurs aux formes de pouvoir et de dépendance inscrites historiquement dans la société.

L'idée de «construction sociale de la réalité » a pu donner lieu, dès lors, à des investigations nouvelles en termes d'instutionnalisation, c'est à dire à des analyses des processus de typification réciproque des actions et des acteurs (P. Berger et T. Luckmann). La cristallisation des habitudes et leur sédimentation dans le temps constituent ainsi des stocks communs de connaissance, dont rend compte le langage et, plus globalement, les institutions et leurs formes de légitimation.

Dès lors se posait, face à ces types d'approches, le problème des conditions sociales de la domination (croyances aux formes de pouvoir légitime) et donc de l'analyse des formes d'interiorisation de celles-ci par les acteurs individuels et collectifs. Des éléments d'explication ont été recherchés soit prioritairement du côté des structures sociales «objectives», économiques ou culturelles (P. Bourdieu dans les années 80), soit en restant attentif aux ambivalences des raisons des acteurs (JC. Passeron et C. Grignon), ou encore en tentent de saisir les propriétés structurelles des systèmes sociaux comme étant à la fois les conditions et les résultats des activités des agents qui font partie de ces systèmes (A. Giddens). Le problème, dans ce dernier cas, est que vouloir considérer ensemble les dimensions de contrainte et de compétence présentées par les structures conduit à des constructions intellectuelles très raffinées mais souvent difficilement susceptibles de mobilisations empiriques.

Ces différentes tentatives indiquent, du moins, chacune à leur manière quel est horizon scientifique vers lequel tendre. Mais leurs difficultés révèlent aussi combien est difficile l'équilibre de funambule que doit respecter le chercheur, pour que le micro acteur ne soit pas finalement privilégié... ou l'inverse.

\section{«LE RETOUR DE L'ACTEUR» DANS LES SCIENCES SOCIALES}

Après une période (forcément noire ...) où la place laissée aux investigations orientées vers les pratiques des acteurs sociaux aurait été laminée par les approches structuralistes et marxistes de l'après-guerre, la conjoncture scientifique serait maintenant essentiellement marquée, aux yeux de certains, par le «retour de l'acteur». C'est là un diagnostic souvent avancé, mais de manière rapide et parfois normative, c'est à dire pour s'en féliciter fort ou au contraire pour le déplorer.

Si l'on veut y voir plus clair et suspendre un moment le jugement, il convient de commencer par s'interroger sur les différentes raisons, dynamiques intellectuelles mais aussi conjonctures politiques, qui auraient pu accompagner voire, même, porter ce «retour de l'acteur» dans les problématiques des sciences 
sociales. En première analyse, on pense ici 1- aux critiques méthodologiques réitérées faites à la posture surplombante du chercheur en sciences sociales, comme seul acteur de l'enquête; 2- mais aussi, à l'accueil aujourd'hui réservé à «l'individualisme méthodologique »dans l'analyse des comportements; 3- sans oublier la réactivation contemporaine des dimensions identitaires des acteurs (individuels ou collectifs); 4- et pour enfin évoquer la valorisation mimétique des acteurs décideurs par les travaux de sciences sociales, qui sont toujours plus en quête de langages partagés avec les commanditaires et financeurs de recherche.

1. Par référence aux sciences naturelles et expérimentales, la posture de l'observateur, de l'enquêteur et du chercheur en sciences sociales a été d'abord conçue comme devant être distante de l'objet d'analyse. Cette distance a été assez facile à envisager dans le cas de réflexions sur des ensembles conceptuels et d'analyse d'agrégats de chiffres; mais la même posture est apparue plus délicate lorsque l'enquête portait sur des études de cas individualisés et des vécus personnels: le problème s'est, par exemple, d'emblée posé pour les savoirs constitutifs de la psychologie, surtout lorsqu'ils sont articulés à la cure et à la clinique.

Pourtant, c'est le modèle du laboratoire, de la distance surplombante à l'objet social et de l'observateur des pratiques quasiment situé« derrière le microscope » qui a durablement prévalu, apparaissant comme la garantie d'une distance à l'ob-

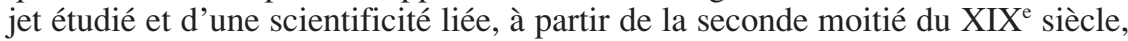
aux formes de l'institutionnalisation universitaire. Mais en définitive, la question n'était pas close; elle a même été ré-alimentée de manière récurrente depuis un siècle par les résultats trop partiels des analyses et par les débats théoriques. L'objet de recherche a fait de la résistance en tant que sujet ... ou plus exactement constitué de sujets d'action. L'acteur résiste à la réification et les pratiques ne se moulent pas que dans des logiques ou des ensembles systémiques. Et du coup, les remise en cause se sont multipliées. L'observateur scientifique, pour sa part, ne doit-il pas faire également partie de la démarche et du champs de l'analyse (variable qui est d'emblée redoutable à contrôler dans la construction et la conduite des protocoles d'enquête par entretiens)?

C'est bien ce type d'interrogations qui semble à présent gagner de plus en plus de disciplines. Mais pour quelles raisons précises et autour de quels enjeux contemporains?

On pourrait ainsi rechercher comment, en réaction autant aux constructions problématiques les plus objectivantes (modèles quantitatifs, enquêtes par questionnaires fermés, analyses comportementalistes) qu'aux «sociologies du soupçon», s'opère dans différents savoirs (sociologie donc, mais aussi géographie sociale, droit réflexif, ou économie régulationniste, par exemple) une re-problématisation de l'acteur, individuel ou collectif. Quels rapports ce mouvement entretient-il avec l'ambition parfois exprimée dans plusieurs démarches de «restituer» les résultats de l'analyse auprès des acteurs enquêtés et observés? Comment démêler (mais le faut-il vraiment?) dans cette ambition ce qui serait de l'ordre de la préoccupation méthodologique (celle qui lie, dans la démarche ethnologique par exemple, l'immersion dans le milieu et la qualité de l'information récoltée) et ce qui relèverait d'une posture quasi morale: rendre, à travers un 
échange à la fois pratique et symbolique) la connaissance élaborée par et pour le scientifique utile aussi à ceux qui ont été« objet» de l'analyse. Il y a-t-il en l'occurrence émergence d'une préoccupation éthique, ou d'un humanisme scientifique nouveau?

2. Il faut aussi considérer par ailleurs, quelle part peut prendre, dans ce supposé «retour de l'acteur», l'influence des problématiques qui s'inspirent, dans différentes disciplines, de «l'individualisme méthodologique» (R. Boudon) et qui valorisent donc, à leur manière, l'acteur individuel?

Par delà la force intrinsèque de cette construction intellectuelle, peut-on y voir également le signe plus général d'une réhabilitation des familles de démarches faisant le pari d'agréger de manière «bottom-up» des observations construites à des échelles individuelles pour, en définitive, espérer rendre intelligible des réalités globales et des caractérisations sociales d'ensemble? Ou bien, serait-ce là plus spécialement le symptôme d'un vaste désarroi théorique, d'une difficulté spécifiquement contemporaine à produire des schémas d'interprétation holistes ou structurels, en nos temps de critique des «grands récits » politico-philosophiques issus du XIX ${ }^{\mathrm{e}}$ siècle?

3. Mais les facettes de ce «retour de l'acteur» dans les sciences sociales sont multiples. Les niveaux de significations ne sauraient se limiter à ce qui précède. On est en effet amené aussi à s'interroger sur la congruence toute particulière des problématiques de l'acteur avec l'actuelle focalisation médiatique et politique qui privilégie à présent la question des constructions identitaires (individuelles et collectives) et des revendications d'appartenance (qu'elles se traduisent en termes d'effets-territoire ou encore d' «invention de la tradition»). Pour aborder les constructions de l'identité et leurs mobilisations psychologiques, économiques ou géo-politiques contemporaines, n'est-il pas précisément opportun (mais donc aussi éventuellement tautologique) de poser les perspectives de l'analyse en termes d'acteur?

4. En dernier lieu, mais ce ne sera pas ici la perspective d'interrogation la moins importante, le thème du «retour de l'acteur» peut nous conduire également à considérer un autre type de «retour», de nature plus réflexive celui-là, qu'il faudrait mener sur les conditions mêmes du financement et de la réception sociale de nos recherches.

En d'autres termes, si aujourd'hui un certain privilège était donné à la thématique de l'action et de l'acteur (individuel et collectif, cette fois), n'est-ce pas que l'accent mis sur ce type de construction de l'objet d'investigation est particulièrement propre à «parler» aux acteurs sociaux eux-mêmes (ceux auxquels s'attachent observations, diagnostics et conseils) et, donc, notamment aux financeurs publics et privés de la recherche, particulièrement soucieux de l'évaluation de l'action publique et de ses acteurs, en y voyant une mesure directe de «l'utilité » des sciences sociales?

On pourrait arguer que ce genre de préoccupations n'est pas précisément neuf, et que l'on peut faire l'histoire des diverses sciences étatiques des populations 
(géographie coloniale, démographie et recensements généraux, criminologie), ou des sciences très liées au développement du marché (statistique, économie d'entreprise), à la gestion de la propriété (droit civil), ou encore au simple «thermomètre » électoral (sondages politiques, enquêtes d'opinion) etc.

Reste que de nouvelles fortunes sociales se sont jouées très récemment, chaque fois qu'une discipline a touché au conseil à l'action et à l'évaluation des pratiques des acteurs sociaux (par exemple, les analyses de l'action stratégique et de la négociation multi-acteurs dans les années 60-70). Par rapport aux activités contemporaines de communication politique qui font une très grande place à l'expertise, et plus généralement par rapport au pilotage de l'action publique (avec le rôle désormais crucial des politiques sanitaires et de prévention des risques majeur), il n'est donc pas indifférent d'interroger «l'utilité» de la thématique de l'acteur et des problématiques qui y sont associées dans le positionnement très contemporain des sciences sociales, de ses catégories de raisonnement et de ses «inventions», telles qu'elles sont mobilisées par les médias et les professionnels de la politique.

Voilà quelques-unes des pistes de réflexion qui avaient été proposées lors de la rencontre de recherche qui a nourri ce numéro de la revue.

Institut d'Etudes politiques

Aix-en-Provence 